\title{
Philosophiques
}

\section{Éthologie et cybernétique : leur approche à la psychologie}

\section{Richard L. Hould et Marc-A. Provost}

Volume 7, numéro 2, octobre 1980

URI : https://id.erudit.org/iderudit/203144ar

DOI : https://doi.org/10.7202/203144ar

Aller au sommaire du numéro

Éditeur(s)

Société de philosophie du Québec

ISSN

0316-2923 (imprimé)

1492-1391 (numérique)

Découvrir la revue

Citer cet article

Hould, R. L. \& Provost, M.-A. (1980). Éthologie et cybernétique : leur approche à la psychologie. Philosophiques, 7(2), 301-319. https://doi.org/10.7202/203144ar d'utilisation que vous pouvez consulter en ligne.

https://apropos.erudit.org/fr/usagers/politique-dutilisation/ 


\title{
ÉTHOLOGIE ET CYBERNÉTIQUE : LEUR APPROCHE À LA PSYCHOLOGIE
}

\author{
par Richard L. Hould et Marc-A. Provost
}

Le Larousse (1973) définit la psychologie comme "l'étude scientifique des faits psychiques"; cette étude serait " devenue aujourd'hui une science en recourant en particulier à la méthode expérimentale, aux statistiques et aux modèles mathématiques". Ces quelques lignes résument bien les aspirations et les contradictions de la psychologie moderne que nous aimerions présenter ici.

\section{A) SCIENCE ET PSYCHISME}

L'étude scientifique des faits psychiques oblige le psychologue à passer par les phénomènes observables. Or, il n'est pas possible d'observer un fait psychique comme tel. Seules ses manifestations peuvent être observées. Confronté à la nécessité d'aborder l'analyse d'un phénomène uniquement par le biais de ses manifestations, le psychologue doit choisir entre trois options qui correspondent chacune à une position théorique et méthodologique.

La première option consiste à redéfinir la psychologie comme l'étude du comportement observable. Watson, dans son Manifeste du behaviorisme (1913), définit la psychologie comme "une branche purement expérimentale des sciences naturelles". Selon lui l'objet exclusif de la psychologie est le COMPORTEMENT défini comme " un ensemble de réactions adaptatives objectivement observables qu'un organisme généralement pourvu d'un système nerveux exécute en riposte à des situations elles aussi objectivement observables ». De la même façon, Wundt, le fondateur du premier laboratoire de psychologie expérimentale, envisage, dès 1879 , la psychologie dans l'optique des sciences de type physico-chimique. Comme le 
fait remarquer Deleule dans son ouvrage la psychologie: mythe scientifique, ces premiers chercheurs croient en la psychologie scientifique en autant que ses techniques expérimentales basées sur le paradigme action-réaction sont analogues à celles de la physique ou de la chimie. Par conséquent, à partir de 1879 , on assiste à une coupure épistémologique d'avec la science antique basée sur la réflexion. En effet, le chercheur en laboratoire manipule des objets, ce qui implique que le savoir est considéré comme le pouvoir que l'on exerce sur ces objets. Il s'agit donc d'oublier les faits psychiques et d'élaborer une science traitant des liens entre les événements et les réactions de l'organisme. Dans le laboratoire, les expériences sont abstraites. En effet, le psychologue offre au sujet des stimuli qui n'ont aucun sens dans la vie quotidienne. Pour MerleauPonty (1942), la conception classique du réflexe, telle que définie par Sherrington, ne représente pas une activité normale de l'animal, puisqu'on l'oblige à répondre à des stimuli isolés, et non à des situations complexes.

L'animal fonctionne donc en « pièces détachées » comme un organisme malade où "les lésions rompent la continuité fonctionnelle des tissus nerveux ». Selon Merleau-Ponty, cette situation du stimulus absolu a deux conséquences: elle entraîne une dissociation pathologique, d'une part, et, d'autre part, elle mène à de nouveaux types d'organisation qui se mettent en place par essai ou erreur. Finalement, MerleauPonty affirme que la notion de stimulus semble aussi confondre l'événement physique tel qu'il est " en soi " et la situation telle qu'elle est pour l'organisme qui, en dernière analyse, est le seul générateur des réactions de l'animal. Il est donc évident que la présentation d'un stimulus absolu n'a aucun sens en soi, puisque la situation expérimentale n'a jamais été vécue et n'est pas naturelle.

Cette prise de position méthodologique qui caractérise les débuts du behaviorisme traditionnel dut, lorsqu'elle sortit du laboratoire pour être appliquée aux personnes, être révisée de façon à accorder une importance de plus en plus grande aux variables cognitives.

Déjà Angell (1906) et ses collaborateurs estiment que la tâche de la psychologie est l'étude du mode de fonctionnement 
qu'utilise l'esprit pour ajuster l'organisme psycho-physique aux changements de son milieu. Pour ce groupe, l'esprit a un rôle qu'on ne peut saisir qu'à travers les actions de l'organisme pris en totalité. Il faut donc, à la fois, effectuer une observation objective des comportements et une introspection minutieuse de la signification qu'accorde le sujet à ces comportements.

Ainsi, la seconde option, la phénoménologie, est née de l'intérêt croissant pour les phénomènes cognitifs. Le phénoménologue considère la psychologie comme étant la science des faits de conscience. Par rapport au comportement observable publiquement, le fait de conscience ne peut être observé que par le sujet lui-même. De plus, les pensées accessibles par l'introspection sont forcément conscientes, alors que le comportement public peut très bien échapper à la conscience du sujet; il suffit qu'un observateur extérieur puisse en prendre note. Le passage de l'étude de la personne comme objet à son étude comme sujet présente des difficultés pour le chercheur qui souhaite adhérer aux méthodes préconisées par les sciences naturelles. En effet, lorsque j'observe mon propre comportement, ou que je porte mon attention sur les sentiments ou les sensations que j'éprouve, ma façon d'agir se modifie. Ces modifications provoquées par l'auto-observation rendent la validité et l'objectivité des données que fournit le sujet sur son expérience privée difficiles à établir ; il faut s'en remettre à l'honnêteté du sujet et prendre pour acquise sa compétence comme observateur. La confrontation des données qu'il fournit avec celles d'observateurs indépendants ne peut être réalisée du fait même qu'il s'agit d'une expérience privée, non observable de l'extérieur.

L'auto-observation de certains automatismes (tension musculaire, tics, etc . . ., peut pousser le psychologue à poser l'hypothèse d'un monde psychique différent de celui de la conscience et du comportement observable. Cette troisième approche rejoint la perspective freudienne où les comportements publics et privés ne sont plus que des manifestations d'une réalité psychique qui échappe à la conscience. Par conséquent, le problème méthodologique demeure entier : comment analyser la structure d'un système uniquement à partir de ce qu'il produit? 
Pour tenter d'apporter un début de solution, nous aimerions maintenant présenter deux nouvelles options qui pourraient s'ajouter aux trois voies classiques que nous venons de présenter : l'éthologie d'une part et la cybernétique d'autre part.

\section{B) ÉTHOLOGIE ET PSYCHOLOGIE}

L'éthologie se rapproche en fait de la première option mentionnée plus haut. Il s'agit, en effet, ici d'observer les liens entre un organisme vivant et son milieu. Cependant, l'éthologie n' « oublie " pas le fait psychique per se ; elle l'infere plutôt à partir de l'observation en le replaçant dans un contexte biologique de l'adaptation au milieu.

L'école éthologique, née de l'intérêt des biologistes pour le comportement animal (Lorenz, Tinbergen, Hinde, EiblEibesfeldt), a, depuis quelques années, fasciné plusieurs chercheurs en psychologie et, plus spécifiquement, les spécialistes du développement humain. Le fait que les divers concepts et méthodes de l'éthologie aient été développés en fonction du désir d'observer des sujets non collaborateurs en milieu naturel (les animaux en liberté) constitue un de ses grands avantages. En effet, il semble possible d'adapter ces méthodes et concepts à l'étude des enfants préverbaux et des enfants inadaptés, qui constituent des groupes d'individus non collaborateurs par excellence (Blurton-Jones, 1972 ; Bowlby, 1969 ; Hutt et Hutt, 1970a, 1970b ; McGrew, 1972).

Comme le mentionne Montagner (1978), l'éthologie veut dépasser les analogies souvent gratuites entre les comportements animaux et les comportements humains. Elle utilise des méthodes connues (l'observation systématique et l'expérimentation) pour étudier efficacement les conduites humaines.

L'éthologie est donc de plus en plus à la mode, mais, comme pour tout système complexe d'explication à la mode, la plupart des gens en ont une idée assez fausse, tout au mieux incomplète.

1) Qu'est-ce que l'éthologie?

Tout le monde s'accorde pour admettre que la définition qu'en a donnée Niko Tinbergen en 1951 est la plus simple et 
la plus acceptable : c'est l'étude biologique du comportement. En effet, l'éthologie considère que la morphologie, la physiologie, le comportement et le milieu naturel d'un individu constituent une entité biologique adaptative dont chaque partie n'est compréhensible qu'en fonction de l'étude de ses relations avec les autres.

En outre, Tinbergen affirme que lorsque l'on pose la question : "Pourquoi l'individu agit-il ainsi »? On pose en fait quatre questions distinctes :

1) Qu'est-ce qui, en ce moment, le fait agir ainsi ? C'est-àdire quelle est la cause du comportement (cause externe environnement ou cause interne - neurophysiologique)?

2) Comment un animal a-t-il grandi pour devenir un animal qui fonctionne ainsi, c'est-à-dire des questions sur le développement et l'apprentissage (l'ontogenèse)?

3) Quelle est l'utilité de ce comportement pour l'animal, c'est-à-dire quelle est la valeur de survie de ce comportement (la fonction)?

4) Pourquoi cette espèce a-t-elle résolu le problème de la survie de cette façon? Et ici nous posons le problème de la phylogenèse ou de l'origine évolutive de cet animal.

Depuis quelques années, une cinquième question s'est rajoutée aux quatre premières : la question de l'organisation du comportement. En effet, comme le souligne Dawkins (1976), le principe de la sélection naturelle nous permet de penser qu'un animal possède un comportement organisé, et ce, même aux plus petits niveaux. Il s'agit donc ici de bien élaborer des schèmes expérimentaux susceptibles de mettre en évidence cette dite organisation. C'est ainsi que, de plus en plus, nous parlons d'analyse hiérarchique du comportement.

La base conceptuelle sur laquelle s'appuient ces questions est la théorie néo-darwinienne de l'évolution : chaque être vivant doit s'adapter à son milieu soit par un trait physique (la fourrure de l'ours polaire), soit par un comportement. Comme le souligne Wilson, une adaptation peut être considérée comme toute caractéristique d'un organisme qui favorise ses chances de reproduction. 
En plus de ces questions, l'éthologie se caractérise nettement par son approche qui insiste sur l'importance de l'observation en milieu naturel comme un prérequis à l'expérimentation. Il faut ici souligner qu'un chercheur qui utilise l'observation comme mode d'approche n'est pas nécessairement un éthologue (Spitz, Iagan, Gesell, etc . . .), s'il ne considère pas ses résultats sous l'angle biologique. Il faut bien garder à l'esprit que l'éthologie se distingue des autres approches en psychologie par son insistance à connaître d'abord le comportement en milieu naturel avant d'expérimenter et par son refus de sélectionner a priori des groupes de comportements. Les hypothèses tirées des observations doivent être vérifiées soit par l'expérimentation, soit par des comparaisons interspécifiques (analogies) qui permettent de dégager les liens de causes et les possibilités d'évolution convergente des espèces comparées.

Ainsi Medawar (1967), dans son livre: The art of the soluble, nous rappelle bien que faire un catalogue du comportement naturel nous permet par la suite d'obtenir de l'information significative à partir de l'étude en laboratoire, car il n'est pas tellement utile d'étudier la variation du comportement à moins de connaitre d'abord la norme à partir de laquelle on peut évaluer cette dite variation.

En résumé, une étude éthologique doit présenter une description exhaustive de la nature et de la fréquence des comportements d'un animal dans son habitat naturel, démontrer une compréhension de la fonction adaptative du comportement et de sa relation avec son milieu, situer le comportement en fonction de sa signification évolutive possible et tenter d'élaborer des hypothèses sur les mécanismes neurophysiologiques sous-jacents (Charlesworth, 1978). Cette image globale du comportement n'implique pas seulement une observation détaillée, mais aussi une interprétation basée sur la théorie néo-darwinienne de l'évolution.

\section{2) L'étbologie bumaine : quelques réalisations}

Comme le souligne Medawar (1976), reconnaître que certaines activités humaines comme le jeu, le cabotinage (showing $u p$ ) et la rivalité sexuelle ne sont pas des innovations 
phylogénétiques dues à notre psyché mais ont plutôt de profondes racines dans l'évolution, jette une lumière nouvelle sur la science du comportement. Quoi qu'il en soit, il semble encore très difficile d'appliquer le cadre théorique de l'éthologie à l'étude de l'espèce humaine ; nous parlerons donc de certaines réalisations puis des problèmes inhérents à l'approche.

Ambrose, en 1968, donne une liste de domaines où « le transfert des idées de la psychologie animale à la psychologie humaine » pourrait être envisagé en soulignant la similarité de phénomènes observés à ces 2 niveaux : le rôle de l'instinct, la communication non verbale, les comportements de déplacement, l'établissement de l'attachement mère-enfant, les périodes sensibles, les stimulations infantiles. On peut ajouter à cette liste l'organisation sociale et les études comportementales en psychiatrie. Nous ne pouvons ici, dans le cadre limité de cet exposé, développer chacun de ces points, mais nous aimerions attirer l'attention sur deux domaines en particulier où l'éthologie a excellé : l'attachement mère-enfant et l'organisation sociale d'enfants d'âge préscolaire.

Le courant de recherches qui se sont centrées sur le développement de l'attachement paraît un exemple de choix. John Bowlby (1969), dans son merveilleux livre Attachment, démontre comment les concepts de l'éthologie peuvent être apprivoisés par le psychanalyste pour tenter de préciser les idées beaucoup trop générales et fort peu opérationnelles de la psychanalyse. Bowlby y démontre combien la notion de survie de l'espèce est évidente lorsque l'on parle d'un nourrisson et combien l'observation systématique du comportement est rentable dans le cadre de la théorie de l'évolution. Nous n'insisterons pas sur le foisonnement de travaux qui ont suivi la parution de ce livre (Ainsworth, 1974; Schaffer, 1971; Sroufe, 1977 ; Lamb, 1976 ; etc . . .). Nous aimerions plutôt souligner en quelques lignes la toute récente fascination que cette relation mère-enfant apporte aux chercheurs qui délaissent peu à peu la seule observation du nourrisson pour se pencher sur l'étude dyadique.

Des chercheurs comme Klauss et Kennell (1975), Daniel Stern (1977), Heideleiss Als et Terry Brazelton (1979), Rudolf 
Schaffer (1977), Hanus Papoušek (1975) ont su mettre en évidence certains comportements types de la mère qui font penser à des stéréotypies comportementales innées (fixed action patterns) que l'on retrouve chez les autres animaux. Bien plus, ils ont commencé à pénétrer dans un monde inconnu jusquelà : la régulation mère-enfant. Tous ces chercheurs ont pu mettre en évidence ce ballet biologique des stimulations mutuelles. Tout se passe comme si la mère et son enfant avaient convenu des limites des stimulations qu'ils pouvaient se donner mutuellement et qu'ils jouaient sur le spectrum d'intensités qu'ils s'étaient donné.

Beaucoup reste à faire dans ce domaine, mais déjà l'importance de la communication enfant-parent pour la survie de l'espèce est pressentie du fait de la précocité même de cette communication. Le deuxième domaine de prédilection de l'éthologie humaine est l'étude des interactions sociales. Des concepts comme affiliation, agression, hiérarchie sociale que l'on retrouve en abondance dans la documentation portant sur les primates (Harlow, 1969 ; Hinde, 1975) ont été repris avec bonheur par quelques éthologistes, tels Blurton-Jones (1972), McGrew (1972), Leach (1972), Strayer (1978) et Montagner (1978), pour étudier la socialisation humaine.

Tous ces chercheurs ont démontré que non seulement la communication non verbale était de prime importance dans le développement du réseau social de l'enfant, mais encore que la plupart des gestes des enfants possédaient une fonction propre. Citons deux exemples : 1) l'agression ritualisée permettrait à un groupe de s'organiser en hiérarchie relativement stable (Strayer, 1978), et 2) la réponse d'un individu serait fonction d'un geste précis et du rang social de l'initiateur (Montagner, 1978).

Comme on peut le constater malgré la rapidité de cet exposé, l'éthologie a su apporter de nouvelles dimensions à l'étude de la psychologie, tout au moins de la psychologie du développement.

Il reste cependant de nombreuses questions à étudier. 


\section{3) Les limites de l'étbologie humaine}

L'éthologie a su démontrer, du moins au niveau de l'enfant d'âge préscolaire, qu'il est normal et rentable de considérer l'homme comme un être biologique (certains disent animal). Cependant, l'espèce humaine pose certains problèmes de taille à l'éthologie, parmi lesquels la médecine, la capacité d'enseigner et d'imiter sont, selon Medawar (1976), les plus importants.

a) la médecine : Nous avons déjà mentionné que le concept central de l'éthologie est l'adaptation définie dans le cadre de la sélection naturelle. Or, au $\mathrm{XX}^{\mathrm{e}}$ siècle, la médecine a fait de tels progrès qu'il est maintenant justifié de dire que la survie de l'homme ne dépend plus exclusivement de l'évolution ou de son héritage biologique. Nous n'en voulons pour preuve que la baisse de la mortalité infantile au cours des 10 dernières années.

b) la capacité d'enseigner et d'imiter : Medawar, en 1972, défendait déjà l'idée que la caractéristique de l'homme n'est pas l'usage d'outils (bomo faber) - distinction qui ne tient plus depuis que nous savons que les primates (Lawick-Goodall, 1971 ; McGrew, 1972) en font autant - mais plutôt la communication de la connaissance d'une génération à l'autre. La caractéristique de cette évolution est donc exogène (de style Lamarckienne), puisqu'elle passe par des canaux non génétiques et en théorie réversible, puisqu'un manque d'éducateurs (cas hypothétique bien sûr) ramènerait rapidement la génération suivante quelques milliers d'années en arrière.

La difficulté n'est donc pas au niveau de la communication même, mais bien au niveau des voies qu'emprunte l'évolution. En ce sens, l'éthologie humaine a beaucoup à faire pour se définir un cadre théorique, et nous croyons que, pour l'instant du moins, elle est fort utile de par sa méthodologie propre et de par sa conceptualisation si l'on s'en tient aux jeunes enfants. 


\section{4) Les avantages méthodologiques}

Peter Dawkins (1976) illustre bien le problème de l'étude du comportement. Il écrit que la compréhension des mécanismes inhérents au comportement impliquerait le diagramme exhaustif des connections du système nerveux d'une espèce, le "Nirvana du neurophysiologiste ». Cependant, selon lui, cette pure réalisation ne nous aiderait probablement pas à bien comprendre comment le comportement fonctionne dans la réalité. Il donne l'exemple de trois ordinateurs ayant le même programme, mais possédant une quincaillerie (hardware) différente - filages, transistors ou circuits intégrés. Ce qui nous intéresse comme utilisateur est nettement plus la réponse de la machine, son software. Et Dawkins ajoute que nous avons besoin d'une explication du software du comportement si nous voulons agir sur ce comportement.

C'est dans ce sens qu'il présente le modèle hiérarchique du comportement. L'idée en soi n'est pas neuve. Cattell et l'analyse factorielle de l'intelligence, Piaget et le structuralisme, Erikson et les phases de développement, Freud et les phases psychosexuelles, et bien d'autres, ont tous tenté de mettre en évidence l'organisation du comportement ou du moins de réduire le comportement humain à un petit nombre de facteurs explicatifs.

L'innovation de l'éthologie est de tenter de mettre en évidence le lien causal et fonctionnel qui existe entre les diverses actions d'une chaîne de comportement. Le rationnel en fait est fort simple. Les facteurs principaux de causes se retrouveront en haut de la classification ou hiérarchie, de sorte qu'il devient possible d'évaluer la partie causale ou fonctionnelle de chaque action en fonction des autres actions du répertoire de l'espèce.

En conclusion, l'éthologie apporte à la psychologie une nouvelle perspective biologique et une prise de conscience de l'importance de l'observation comme prérequis à toute forme d'expérimentation. Elle permet en outre, comme le souligne Medawar (1976), de libérer le chercheur du besoin de trouver une explication causale à chaque phénomène observé et de la tyrannie doctrinaire d'un système d'explication déjà existant. 


\section{C) CYBERNÉTIQUE ET PSYCHOLOGIE}

Tiraillés entre les limites trop vite atteintes d'une approche qui s'astreint à ne considérer que le comportement de la personne et le souci de respecter les contraintes qu'impose la méthode scientifique à l'ensemble des sciences de la nature, des psychanalystes (Peterfreund et Schwartz, 1971), des behavioristes (Goldfried et Davidson, 1976) et des thérapeutes centrés sur le client (Wexler et Rice, 1974) persistent à vouloir faire de l'étude des faits psychologiques une science au même titre que les sciences de la nature. Cependant, pour se dégager de l'impasse où les méthodes de la physique traditionnelle plongent la psychologie (voir plus haut), ils se tournent vers la cybernétique et l'importance qu'elle accorde à l'information et à son traitement. Piaget (1970) affirme que "la tendance actuelle la plus générale est naturellement orientée vers les modèles cybernétiques ou de simulation des activités mentales de tout genre impliquant des régulations, spécialement dans le domaine des activités supérieures " (p. 221). Il ajoute que "le grand avantage d'un tel modèle est de permettre une analyse des opérations constitutives et non pas seulement des résultats ou performances comme c'est souvent le cas d'autres modèles " (p. 222). La cybernétique permet au psychologue d'aborder l'étude des phénomènes dans toute leur complexité.

Pour Buckley (1968), l'étude du comportement humain reste le domaine des sciences qui a le moins répondu au défi stimulant qu'offre la perspective des systèmes modernes. Ce serait pourtant la partie des sciences qui a le plus à gagner des découvertes et des travaux concernant l'analyse des systèmes et des machines qui traitent de l'information. D'après Martin (1976), l'enthousiasme vers le milieu des années 60 pour la théorie générale des systèmes se serait estompée à cause de la difficulté qu'implique la conceptualisation des émotions et des cognitions dans le cadre de la théorie des systèmes et de la crainte qu'une telle conceptualisation ne réduise la personne en quelque chose de mécanique et de non humain. Mais, l'homme reste ce qu'il est peu importe la conceptualisation qu'on en fasse. En attendant, cette approche suggère une méthode de recherche qui, loin de rejeter les méthodologies traditionnelles, s'y ajoute en les intégrant et en les raffinant. Elle consiste à 
construire un modèle de traitement d'information et à le simuler sur ordinateur.

\section{1) Structure interne et simulation}

La stratégie de recherche suggérée par la théorie des systèmes consiste à élaborer une théorie de la structure interne de la personnalité du sujet permettant de relier les propriétés de la situation où il se trouve aux réactions et aux comportements qu'il présente. Cette théorie aboutit à la construction d'une machine, à une simulation sur ordinateur ou simplement à une analyse par des formules mathématiques. Pour Arbib (1972), celui qui comprend un phénomène peut construire une machine qui l'imite. Le problème méthodologique majeur de cette stratégie de recherche consiste à déterminer les opérations qui vont permettre de reproduire les transformations d'une situation particulière en un comportement spécifique. Le comportement et la situation étant observables, on ne peut qu'inférer les variables latentes inhérentes au fonctionnement psychologique de la personne (Gréco, 1967). Cette méthode repose sur l'exploitation systématique de l'analogie entre les propriétés d'un organisme naturel et celles d'un algorithme artificiel construit en laboratoire ou sur papier. Le critère de vérité de la science traditionnelle est remplacé par le critère de similitude ou d'adéquation du modèle au réel. Dans cette perspective, l'objet de la recherche psychologique est de construire un modèle qui fournisse une représentation des processus par lesquels la personne traite l'information qui lui est disponible à un moment donné.

Le traitement de l'information concerne directement la création des significations. En effet, c'est à partir de l'analyse plus ou moins consciente de l'ensemble des informations dont elle dispose que la personne peut dégager les significations qui déterminent son vécu émotif et comportemental. Tout comme le programme de l'ordinateur, la pensée humaine peut être considérée comme une suite d'étapes par lesquelles les informations sont sélectionnées, élaborées et réorganisées. À chaque étape du traitement de l'information, un induit se transforme en un produit qui, à son tour, devient une partie de l'étape suivante et ainsi de suite jusqu'au produit final du système de 
traitement. Chacune des étapes de la hiérarchie du traitement de l'information se caractérise par des perceptions et des comportements spécifiques. Ainsi, la perception d'une couleur, d'un objet, d'un mouvement, d'une relation, d'une abstraction reflètent autant de niveaux divers dans la hiérarchie des systèmes de traitement d'information (Powers, 1973).

\section{2) La simulation de processus psycbologiques}

Pour comprendre les programmes de traitement d'information d'une personne, le chercheur doit prendre une attitude de clinicien. Il observe ce qui se passe dans une situation en tâchant d'adopter le point de vue du sujet. Il tente de percevoir la situation telle que vécue subjectivement par la personne en situation. Que perçoit le sujet dans son environnement? Quels sont les éléments qui attirent son attention ? Comment organise-t-il son monde? À quels choix est-il confronté ? Comment l'information disponible se transforme-t-elle en une décision particulière? Quel serait le programme d'une machine qui, à partir des informations disponibles au sujet, aboutirait à la même décision, à la même perception, au même comportement que lui ? D'après Jackson (1974), l'introspection constitue probablement la source d'information la plus communément utilisée en intelligence artificielle pour la recherche sur les processus spécifiquement humains de traitement d'information. S'ajoutent à cette observation les données que fournit la science au chercheur.

La deuxième étape de la simulation consiste à élaborer un modèle qui fournit une représentation du traitement de l'information qui se déroule à l'intérieur de la pensée de la personne confrontée à une situation donnée. Ce modèle permet d'expliciter le cheminement par lequel une information acquiert une signification. Ce cheminement s'exprime sous la forme d'un programme. Chaque étape du programme contient les règles par lesquelles les informations d'un niveau donné se transforment en significations d'un niveau hiérarchiquement supérieur. Les significations produites par un niveau de traitement peuvent servir d'induit au niveau de traitement subséquent. Habituellement, la personne n'a pas conscience de l'activité et des transformations qu'effectue son système ner- 
veux. Elle ne perçoit que les conséquences de ces transformations. Alors que les conséquences de la transformation peuvent être associées à une image perceptuelle, les processus de transformation correspondent probablement à une pensée sans image. Le chercheur peut, grâce à l'introspection, explorer l'ensemble des significations des divers niveaux d'un processus perceptuel donné. Cet ensemble de significations constituerait le vécu expérientiel du sujet. Le chercheur ne peut qu'inférer la nature des règles de transformation qui relient ces significations entre elles.

Le psychologue peut utiliser la simulation pour vérifier la théorie qu'il propose sur ce qui se passe dans le cerveau de la personne confrontée à une situation. La troisième étape de la recherche consiste à programmer un robot ou une machine de façon que sa réaction à une situation donnée s'apparente à celle de la personne étudiée. La simulation implique qu'à partir des données brutes disponibles au sujet, la machine aboutisse aux mêmes décisions que ce dernier. Que l'information soit traitée par des neurones ou des circuits électroniques, l'intérêt de la recherche porte sur la logique ou la programmation ; les détails techniques peuvent être abandonnés aux spécialistes des autres disciplines ou même aux psychologues intéressés à la physiologie du cerveau humain. La tâche du psychologue qui utilise la simulation comme instrument de recherche consiste à transposer la théorie qu'il a développée en un algorithme susceptible d'être ensuite programmé dans les circuits de la machine. Il suffit que les opérations mathématiques ou algébriques du modèle artificiel constituent une approximation des opérations mentales effectuées par le cerveau humain. Dans cette perspective, l'objectif de la recherche en psychologie est de fournir la meilleure approximation du fonctionnement de la pensée. La similitude entre la réaction du robot et celle du système naturel peut être mesurée et vérifiée empiriquement. Le réductionnisme de la simulation est un réductionnisme conscient par lequel la complexité et le merveilleux de la pensée humaine se révèlent.

Que le modèle constitue une bonne ou une mauvaise estimation du système naturel concerne la validité même du modèle artificiel. L'étude de la validité du modèle à travers la 
validité hypothético-déductive des mesures qu'il produit constitue la dernière étape de la recherche par simulation. Après avoir construit son modèle et recueilli les informations qui l'alimentent, la tâche du chercheur consiste à comparer les décisions de la machine aux décisions de la personne. L'analyse des divergences entre ces deux types de décision permet d'évaluer et d'améliorer le programme de simulation et, par le fait même, notre compréhension du fonctionnement de la personne. La validation de ces divers construits peut s'effectuer directement auprès du sujet en lui demandant si les valeurs fournies par le programme de simulation correspondent aux divers aspects de son propre fonctionnement. $\grave{A}$ cette première validation peut s'ajouter la comparaison entre des groupes d'individus caractérisés par une décision ou une attitude particulière face à la problématique étudiée.

En plus de rendre possible une étude objective de la subjectivité humaine, ce type de recherche aide le théoricien à éviter les pièges de la réification des fonctions et de l'anthropomorphisme. En effet, cette approche exige l'explicitation détaillée du processus simulé et la définition opérationnelle de chacun des construits constituant le modèle. En conséquence, le programme de simulation devient un instrument de mesure des divers aspects du problème étudié, et le psychologue peut mettre en valeur ses talents de théoricien et de psychométricien.

\section{3) Conclusion}

L'un des auteurs de cet article a eu l'occasion d'utiliser cette méthodologie de recherche dans un ouvrage intitulé : «Perception interpersonnelle et entente conjugale. Simulation d'un système » (Hould, 1979). Ce travail portait sur l'élaboration et la validation d'un ensemble de construits concernant les processus impliqués lors de la perception interpersonnelle. L'agencement entre ces construits correspondait à un modèle qui permit d'intégrer la théorie des répertoires de comportements interpersonnels de Leary (1957), les notions de symétrie et de complémentarité des relations interpersonnelles de Watzlawick et al (1967), le concept de coût des relations de Homans (1958) de même que la conception de la satisfaction 
et de la dépendance dans le cadre de la théorie de l'échange social de Thibaut et Kelley (1959). Des mesures de la complexité cognitive de Kelly (1955) et de la cohérence sémantique (Benjamin, 1974) se sont ajoutées à toutes les variables précédentes. Treize de ces vingt-neuf variables se sont avérées reliées avec le degré de fonctionnement du sujet à l'intérieur du couple.

Ce type de recherche peut être très fécond tant aux plans théorique, empirique que pratique. Au plan conceptuel, la recherche aboutit à un modèle qui permet une représentation des processus qui, par une succession de niveaux de conscience, transforment, dans l'application citée plus haut (Hould, 1979), la perception d'un ensemble de comportements interpersonnels en une attitude de la personne à l'égard du couple qu'il forme avec un partenaire spécifique. La personne, comme le modèle, peut être conçue comme un canal de transmission d'informations qui a pour induit les mémoires de comportements interpersonnels associés à des personnages clés de la vie du sujet, et, pour produit, la disponibilité du sujet à modifier son attitude pour maintenir l'existence du couple qu'il forme avec son partenaire. Cette théorie, représentée par le modèle, est ensuite confrontée à des critères extérieurs de validation. Cette confrontation fournit une abondance de données empiriques qui permettent de valider le modèle, de le remettre en question ou encore de fournir un nouvel éclairage sur la nature des phénomènes étudiés. Finalement, la recherche produit un instrument de mesure susceptible de générer d'autres recherches et de présenter une utilité pratique certaine.

\section{D) CONCLUSION GÉNÉRALE}

En conclusion, l'éthologie et la cybernétique semblent deux avenues fort prometteuses pour la recherche en psychologie. Toutes deux partent de considérations historiques visant à mettre en lumière les énormes problèmes que la psychologie expérimentale classique pose, et toutes deux tentent d'élaborer des concepts englobant à la fois l'homme, son comportement et son milieu en un tout cohérent.

Département de psychologie

Université du Québec à Trois-Rivières 


\section{RÉFÉRENCES BIBLIOGRAPHIQUES}

AINSWORTH, M. (1974). "The development of infant-mother attachment", in B.M. Caldwell and H.N. Ricciuti (Eds.) : Review of child development research, 3. Chicago : University of Chicago Press.

ALS, H. \& BRAZELTON, T. (1979). "Social interaction : dynamic matrix for developing behavioral organization", in J. Uzgiris (Ed.) : Social interaction and communication during infancy, 21-39. San Francisco : Jossey-Bass.

AMBROSE, A. (1968). "The comparative approach to early child development : the data of ethology", in E. Miller (Ed.) : Foundations of child psychiatry. Oxford : Pergamon.

ANGELL, J.R. (1906). The province of functional psychology. Discours présidentiel à l'American Psychology Association.

ARBIB, M.A. (1972). The metaphorical brain. New York: WileyInterscience.

BENJAMIN, L.S. (1974). "Structural analysis of social behavior". Psychological review, $81,392-425$.

BLURTON-JONES, N. (1972). Etbological studies of child behaviour. Cambridge: University Press.

BOWLBY, J. (1969). Attacbment and loss. Vol. 1. Attacbment. London : Hogarth Press.

BUCKLEY, W. (1968). "Society as a complex adaptive system", in W. Buckley (Ed.): Modern systems research for the bebavioral scientist. Chicago : Aldine.

CHARLESWORTH, W. (1978). "Ethology : its relevance for observational studies of human adaptation", in G.P. Sackett (Ed.) : Observing behavior. Baltimore : University Park Press.

DAWKINS, R. (1976). "Hierarchical organization: a candidate principle for ethology", in P.P.G. Bateson \& R.A. Hinde (Eds.): Growing points in etbology. Cambridge : Cambridge University Press.

DELEULE, D. (1969). La psychologie, mythe scientifique. Paris : Robert Laffont.

GOLDFRIED, M.R., DAVISON, G.C. (1976). Clinical behavior therapy. Montreal : Holt, Rinehart \& Winston.

GRECO, P. (1967). "Épistémologie de la psychologie ", in J. Piaget (Ed.) : Logique et connaissance scientifique. Dijon: Encyclopédie de la pléiade, 22.

HARLOW, H.F. (1969). "Age-mate or peer affectional system". Advance study of behaviour, 2, 333-383.

HINDE, R.A. (1975). "Interactions, relationships and social structure". Man, $I I, 1-17$.

HOMANS, G.C. (1958). "Social behavior as exchange". American journal of sociology, 63, 597-606. 
HOULD, R. (1979). Perception interpersonnelle et entente conjugale. Simulation d'un système. Thèse de doctorat inédite: Université de Montréal. Copies disponibles auprès de l'auteur, Département de psychologie, U.Q.T.R.

HUTT, S.J. et HUTT, C. (Eds.) (1970a). Behaviour studies in psychiatry. Oxford : Pergamon.

HUTT, S.J. et HUTT, C. (1970b). Direct observation and measurement of behavior. Springfield, Illinois : Charles Thomas.

JACKSON, P.C. (1974). Introduction to artificial intelligence. New York: Petrocelli.

KELLY, G.A. (1955). The psychology of personal construct. New York : Norton.

KLAUS, M.H. \& KENNELL, J.H. (1975). Maternal-infant bounding. St-Louis : Mosby.

LAMB, M. (1976). The role of the fatber in child development. New York: Wiley.

LAWICK-GOODALL, J.V. (1971). In the shadow of man. Boston: Hougton Mifflin.

LEACH, G.M. (1972). "A comparison of the social behaviour of some normal and problem children", in N. Blurton-Jones (Ed.): Ethological studies of child behaviour, 249-284. Cambridge: Cambridge University Press.

LEARY, T. (1957). Interpersonal diagnosis of personality. New York : Ronald.

MARTIN, P.A. (1976). A marital therapy manual. New York : Brunner/ Mazel.

MCGREW, W.C. (1972). A ethological study of children's bebaviour. New York : Academic Press.

MEDAWAR, P.B. (1967). The art of the soluble. London : Mcthuen.

MEDAWAR, P.B. (1972). "Technology and evolution", in Technology and the frontiers of knowledge. The Frank Nelson Doubleday lectures. 1972-1973. New York : Doubleday.

MEDAWAR, P.B. (1976). "Does ethology throw any light on human behaviour", in P.P.G. Babeson \& R.A. Hinde (Eds.): Growing points in ethology. Cambridge : Cambridge University Press, 497-506.

MERLEAU-PONTY, M. (1942). La structure du comportement. Paris : P.U.F.

MONTAGNER, H. (1978). L'enfant et la communication. Paris : Stock.

PAPOUŠEK, H. \& PAPOUŠEK, M. (1975). "Cognitive aspects of preverbal social interaction between human infants and adults", in Parent-infant interaction, Ciba foundation Symposium 33. Amsterdam : Elsevier.

PETERFREUND, E., SCHWARTZ, J.T. (1971). Information, systems, and psycho-analysis. New York : International University Press.

PIAGET, J. (1970). Épistémologie des sciences de l'bomme. Paris : Gallimard.

POWERS, W.T. (1973). Bebavior: the control of perception. Chicago: Aldine. 
SCHAFFER, H.R. (1971). The growth of sociability. Penguin Science of behavior.

SCHAFFER, H.R. (1977) (Ed.). Studies in motber-infant interaction. London : Academic Press.

SROUFE, L.A. (1977). "Wariness of strangers and the study of infant development". Child development, Vol. 48, 731-746.

STERN, D. (1977). The first relationship : infant and mother. London : Open Books.

STRAYER, F.F. (1978). "L'organisation sociale chez les enfants d'âge préscolaire ". Sociologie et société, 10, 43-64.

THIBAUT, J.W., KELLEY, H.H. (1959). The social psychology of groups. New York: Wiley.

TINBERGEN, N. (1951). L'étude de l'instinct. Paris : Payot.

WATSON, J.B. (1913). "Psychology as the behaviorist views it". Psychological review, 20, 158-177.

WATZLAWICK, P., BEAVIN, J.H. JACKSON, D.D. (1967). Une logique de la communication. Paris : Éditions du Seuil, 1972.

WEXLER, D.A., RICE, L.N. (1974). Innovations in client-centered therapy. Toronto : John Wiley.

WILSON, E.O. (1975). Sociobiology: the new synthesis. Cambridge, Massachusetts : The Belknap press of Harvard University Press. 